\title{
Specialized mouse embryonic stem cells for studying vascular development
}

This article was published in the following Dove Press journal:

Stem Cells and Cloning:Advances and Applications

7 October 2014

Number of times this article has been viewed

\author{
Drew E Glaser' \\ Andrew B Burns ${ }^{2}$ \\ Rachel Hatano ${ }^{2}$ \\ Magdalena Medrzycki ${ }^{3}$ \\ Yuhong Fan ${ }^{3}$ \\ Kara E McCloskey' \\ 'School of Engineering, University of \\ California, Merced, CA, USA; ${ }^{2}$ School \\ of Natural Sciences, University of \\ California, Merced, CA, USA; ${ }^{3}$ School \\ of Biology and the Petit Institute \\ for Bioengineering and Bioscience, \\ Georgia Institute of Technology, \\ Atlanta, GA, USA
}

\begin{abstract}
Vascular progenitor cells are desirable in a variety of therapeutic strategies; however, the lineage commitment of endothelial and smooth muscle cell from a common progenitor is not well-understood. Here, we report the generation of the first dual reporter mouse embryonic stem cell (mESC) lines designed to facilitate the study of vascular endothelial and smooth muscle development in vitro. These mESC lines express green fluorescent protein (GFP) under the endothelial promoter, Tie-2, and Discomsoma sp. red fluorescent protein (RFP) under the promoter for alpha-smooth muscle actin ( $\alpha$-SMA). The lines were then characterized for morphology, marker expression, and pluripotency. The mESC colonies were found to exhibit dome-shaped morphology, alkaline phosphotase activity, as well as expression of Oct 3/4 and stage-specific embryonic antigen-1. The mESC colonies were also found to display normal karyotypes and are able to generate cells from all three germ layers, verifying pluripotency. Tissue staining confirmed the coexpression of VE (vascular endothelial)-cadherin with the Tie-2 GFP+ expression on endothelial structures and smooth muscle myosin heavy chain with the $\alpha$-SMA RFP+ smooth muscle cells. Lastly, it was verified that the developing mESC do express Tie-2 GFP+ and $\alpha$-SMA RFP+ cells during differentiation and that the GFP+ cells colocalize with the vascular-like structures surrounded by $\alpha$-SMA-RFP cells. These dual reporter vascular-specific mESC permit visualization and cell tracking of individual endothelial and smooth muscle cells over time and in multiple dimensions, a powerful new tool for studying vascular development in real time.
\end{abstract}

Keywords: vascular progenitor cells, endothelial cells, smooth muscle cells, embryoid body, vasculogenesis

\section{Introduction}

Embryonic stem cells (ESC) are pluripotent cells that boast unlimited expansion potential in vitro and are able to differentiate into cells from all three germ layers. ${ }^{1}$ Because ESC can be easily maintained and expanded in tissue culture systems, they are an excellent in vitro model for studying development and stem cell fate and as a cell source in cell transplantation therapies. Vascular progenitor cells are desirable in therapeutic strategies for patients exhibiting ischemic vascular disease, ${ }^{2}$ for coating of vascular grafts to inhibit platelet adhesion and clotting, ${ }^{3,4}$ and as vehicles for gene therapy as well as various tissue engineering applications. ${ }^{5}$

In the yolk sac of mammals, hematopoietic and endothelial lineage precursors differentiate into blood islands. The outer cells of the blood islands are endothelial, whereas the inner cells give rise to haematopoietic progenitors. These cells later become the primary vascular plexus. Within the embryo, angioblastic precursors aggregate into solid strands forming the ventral and dorsal aortas and vitelline arteries and veins. These
Correspondence: Kara E McCloskey School of Engineering, University of California, Merced, PO Box 2039,

Merced, CA, 95344, USA

Tel + I 2092287885

Fax +I 2092284053

Email kmccloskey@ucmerced.edu submit your manuscript | www.dovepress.com

Dovepress

http://dx.doi.org// 0.2147/SCCAA.S69554
Stem Cells and Cloning:Advances and Applications 2014:7 79-88 
vascular systems are further remodeled through sprouting (angiogenesis), branching, and intussusception from existing blood vessels and specialize as arteries, veins, or capillaries (reviewed $\mathrm{in}^{6}$ ). Attempts to study vasculogenesis have led to the identification of a receptor for vascular endothelial growth factor (VEGF), called Flk-1, that is expressed on lateral plate mesodermal cells. ${ }^{7}$ Flk-1 is well-known as a prominent early differentiation marker for both endothelial cell (EC) and blood cells in the developing embryo and embryonic stem cell cultures. $^{8-11}$

The development of EC and vascular smooth muscle cells (SMC) from a common Flk-1+ expressing progenitor cell has been previously established ${ }^{12}$ in a groundbreaking study. This work also showed that VEGF promoted EC differentiation while mural cells were induced by platelet-derived growth factor-BB and not by transforming growth factor-beta. However, despite a number of subsequent reports on the roles of various growth factors ${ }^{12-14}$ and extracellular matrix components $^{15,16}$ in the specification of vascular cell fate, the complete signaling and regulatory mechanisms at the various stages of vascular cell induction remain incomplete.

Therefore, in order to better study vascular development in real time, we set out to develop a unique embryonic stem cell line that would express fluorescent proteins corresponding with later stage SMC and EC commitment. We expect that this cell line will allow unique real time investigations of cell fate from single vascular progenitor cells as well as morphological development of blood vessels. We chose to use alpha-smooth muscle actin ( $\alpha$-SMA) as the molecular marker for SMC and Tie-2 as the marker for endothelial cells. Tie-2 is tyrosine kinase cell surface receptor that is activated by angiopoeitin binding. Tie- 2 is present on both vascular EC and some hematopoietic cells ${ }^{17}$ and plays a role in regulating endothelial cell survival and blood vessel maturation. $^{18}$

The transgenic mouse expressing RFP under the $\alpha$-SMA promoter was developed by Dr David Allen Brenner ${ }^{19}$ and kindly donated for the generation of the dual fluorescent line. A transgenic mouse expressing GFP under the Tie-2 promoter was generated by Motoike et $\mathrm{al}^{20}$ and purchased from Jackson Laboratories. The mice were bred and the blastocysts were isolated for the generation of the dual reporting vascular ESC lines. These dual reporter lines permit the visualization of individual endothelial and smooth muscle cells within a population while also facilitating the temporal tracking of individual cells during morphological development.

\section{Methods \\ Generation of embryos}

This protocol was approved by the Institutional Animal Care and Use Committee (IACUC) of the University of California, Merced (Animal Welfare Assurance \#A4561-01). A female mouse expressing GFP under Tie-2, (Tie-2 GFP; Jackson Laboratories) was mated with a male mouse expressing the Discomsoma sp. RFP under $\alpha$-SMA RFP (from Dr Brenner, University of California, San Diego) and observed for a postcoital (pc) plug the following morning (Figure 1). The presence of a plug was considered day 0 of embryo development. We then treated the pregnant mouse in order to delay the implantation (up to 8 days pc) of the developing blastocysts using modified methods from previously published protocols. ${ }^{21-23}$ On day $2 \mathrm{pc}$, the pregnant female mouse was given an intraperitoneal injection of $10 \mu \mathrm{g}$ of Tamoxifen (Sigma) dissolved in propylene glycol and a subcutaneous injection of $3 \mathrm{mg}$ of Depo-Provera (Sigma) dissolved in phosphate buffered solution (PBS).

\section{Extraction and isolation of the ESC}

On day 7 pc, the female mouse was sacrificed by cervical dislocation. The uterine horns were removed and flushed with PBS and the blastocysts were collected (Figure 1) and plated in separate wells of a 12-well plate on mouse embryonic fibroblasts (MEF). The cells were initially fed ESC Culture Medium consisting of Knockout Dulbecco's Modified Eagle's Medium (KO-DMEM) (Invitrogen) supplemented with $15 \%$ fetal bovine serum (Invitrogen), 5\% Knockout Serum Replacement (KSR) (Invitrogen), 1x nonessential amino acids (Invitrogen), 1x penicillin streptomycin, 1x L-glutamine, $0.1 \mathrm{mM}$ 2-mercaptoethanol (Calbiochem), and 1,000 units per $50 \mathrm{~mL}$ of leukemia inhibitory factor (Chemicon). After the first several passages, the cells were cultured in serum-free medium consisting of KO-DMEM (Invitrogen) supplemented with 15\% KSR (Invitrogen), 1x nonessential amino acids (Invitrogen), 1x penicillin streptomycin, 1x L-glutamine, $0.1 \mathrm{mM}$ 2-mercaptoethanol (Calbiochem) and 2,000 units $/ \mathrm{mL}$ of leukemia inhibitory factor (Chemicon), and $10 \mathrm{ng} / \mathrm{mL}$ of bone morphogenetic protein-4 (R\&D Systems). The morphology of the hatching blastocysts and the expansion of the inner cell mass (ICM) was observed each day for 4 days (Figure 1). The zona pellucida is visible during day 0 and day 1 before the blastocyst firmly attaches to the MEF layer. After attachment at day 2 , the trophoectoderm peeled away and the developing ICM became more prominent. After 4 days, the hatched blastocysts were flooded with $0.5 x$ trypsin (Invitrogen) in 


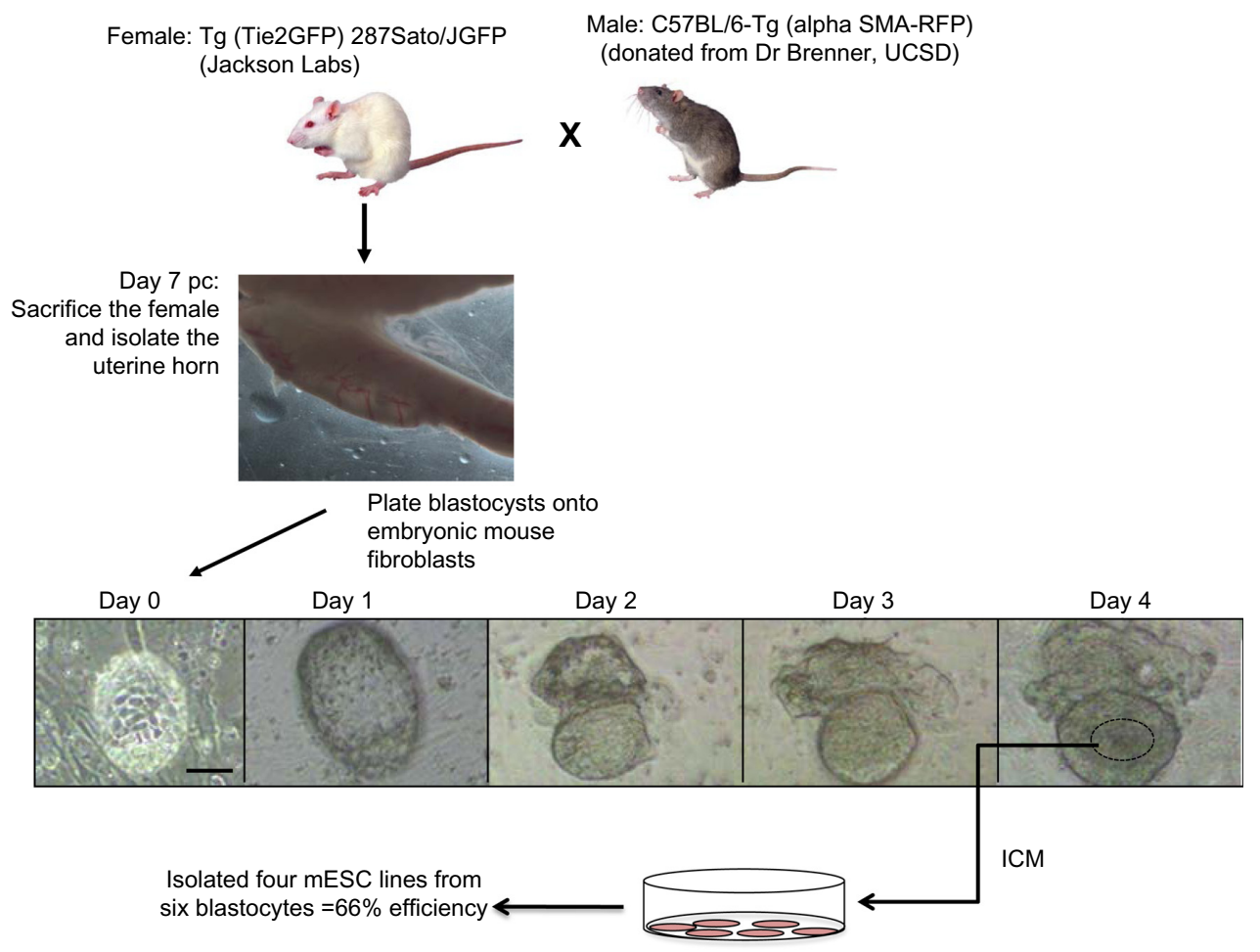

Figure I Flow chart of methodology for establishing the new mESC lines.

Notes: On day 0 , the mice were bred together, and pregnancy was verified the following day by the presence of plug. At day 2 , the implantation of embryo was delayed by injecting a pregnant female with $10 \mu \mathrm{g}$ tamoxifen and $3 \mathrm{mg}$ of Depo-Provera. After 7 days, the pregnant female was euthanized. The delayed blastocysts were then isolated, by flushing uterine horn, and plated on embryonic fibroblast feeder cells. From days 7-II (days $0-4$ after plating the blastocysts), each blastocyst hatched to reveal its ICM. Images of the blastocysts after plating include a collected blastocyst at day 0 , the blastocyst attached to the supportive fibroblast feeder layer with zona pellucida at day I, the trophoectoderm beginning to peel away from the blastocyst at day 2, and the ICM developing from days $2-4$. By day 4 , the ICM was developed enough to be manually selected from each hatching blastocyst, disassociated into individual cells, and replated. Scale $=50 \mu \mathrm{m}$.

Abbreviations: mESC, mouse embryonic stem cell; ICM, inner cell mass; SMA, smooth muscle actin; RFP, red fluorescence protein; UCSD, University of California, San Diego; pc, post-coital.

order to disaggregate the ICM. Once the ICM loosened into nonadherent cells (approximately 5-10 minutes of attentive observation under microscope), the solution was neutralized with ESC Culture Medium. The loosened ICM cells from each blastocyst were manually picked with an aspirator pipette system and transferred to individual MEF-coated plates and fed ESC Culture Medium. The developing ESC colonies were observed over the next 10 days with half medium changes occurring approximately every 4 days. After 10 days, the cells were maintained and expanded on MEF-coated dishes with full medium changes every other day. From one pregnant mouse, four lines of ESC were generated. Here we characterize two of the lines, named A3 and B2.

\section{Karyotype analysis}

ESC were cultured on mitotically inactivated mouse embryonic fibroblasts feeder layers and subsequently cultured on feeder-free $0.1 \%$ geletin-treated dishes prior to being treated with $10 \mathrm{uL} / \mathrm{mL}$ Karyo-MAX colcemid (Gibco) for 60 minutes. ESC were washed with PBS, trypsinized, and harvested. ESC pellets were resuspended in hypotonic solution $(75 \mathrm{mM} \mathrm{KCl})$ for 7 minutes at $37^{\circ} \mathrm{C}$, followed by fixation with Carnoy's solution (3 volumes of methanol to 1 volume of acetic acid) for 30 minutes. Fixed cells were concentrated and dropped onto an angled, humidified microscope slide. The slide was washed with $1 \mathrm{~mL}$ of fixation solution to clean up the debris and subsequently dried. The chromosomes were stained with Hoechst dye (1:1,000 dilution) for 60 minutes at room temperature in the dark and imaged with an Olympus fluorescence microscope.

\section{Characterization of ESC}

The ESC-like colonies were analyzed for 3-D morphology on MEF layers. Cells were also treated with $0.7 \%$ Triton X-100 (MP Biomedicals, LLC) to permeabilize the cells and stained with alkaline phosphatase (Cell Biolabs) per the manufacturer's instructions. Images of stained cells were taken with an inverted phase contrast microscope. ESC-A3 (passage \#12) and ESC-B2 (passages \#11) were also plated in a twelve-well dish coated with MEF at a concentration of 10,000 cells 
per well. ESC were allowed to establish well-defined colonies. After 4 days, the cells were fixed with 4\% paraformaldehyde and stained with rabbit anti-mouse Nanog (Cell Signalling) and Sox 2 (Abcam), biotinylated stage-specific embryonic antigen-1, and Oct3/4 PE. The primary antibodies were allowed to incubate overnight in PBS with $1 \mathrm{mg} / \mathrm{mL}$ BSA, $5 \%$ donkey serum, $0.7 \%$ triton $\mathrm{x}-100$ (except the biotinylated antibody) and counterstained with donkey anti-rabbit FITC (Abcam) for Sox-2, donkey anti-rabbit Alexaflour 488 (Invitrogen) for Nanog, or streptavidin FITC (Fitzgerald) for approximately 2 hours at room temperature. Cells were visualized using either a Nikon TE 2000 fluorescent microscope (Technical Instruments) or a Laser Scanning Confocal Microscope (Technical Instruments).

\section{Tumor assay for pluripotency}

Severely immune-compromised NOD/SCID IL2 receptor gamma chain knockout (NSG) mice (Jackson Laboratories) were injected with 1 million ESC in $100 \mu \mathrm{L}$ of PBS subcutaneously in the dorsal flanks of each mouse using a 28 gauge needle. After 30 days, the mice were euthanized by cervical dislocation and the teratomas harvested and sent out for histological processing (IDEXX Laboratories). The prepared slides were then dehydrated in graded alcohol, de-paraffinized using three washes of xylene, then rehydrated in graded alcohol and placed in PBS until imaging with a confocal microscope. After deparaffinization, the GFP and RFP were either directly visualized, or counterstained in 1\% BSA (Sigma), 5\% donkey serum (Fitzgerald), 0.7\% Triton X-100 (MP Biomedical) followed by a goat anti-DsRed antibody (Santa Cruz, 1:50 dilution) followed by donkey-anti goat PE (Abcam, 1:100 dilution). DAPI (4',6-Diamidino-2-Phenylindole, Dilactate) was used to visualize the nuclei and images were taken on a Nikon TE 2000 fluorescent microscope. To verify endothelial and smooth muscle cell identity, goat anti-VE (vascular endothelial)-cadherin (Santa Cruz, 1:100) with a donkey antigoat (Santa Cruz, 1:100) or rabbit anti-smooth muscle myosin heavy chain (Abcam, 1:50) with a donkey anti-rabbit FITC (Abcam, 1:50) was also used in conjunction with the direct visualization of the GFP or the counter stained RFP.

\section{Analysis of 3D sprouting vascular-like structures}

ESC were plated in tissue culture plates to deplete the MEF population as described above, then resuspended in medium consisting of alpha-Minimal Essential Medium (Cellgro), $20 \%$ fetal bovine serum, $1 \mathrm{x}$ penicillin-streptomycin, and
$50 \mu \mathrm{g} / \mathrm{mL}$ of VEGF (R\&D Systems). The mESC were then allowed to aggregate into embryoid bodies (EB) at approximately 1,000 cells per aggregate using the hanging drop method. After 1 day, the mESC aggregates were rinsed into a $60 \mathrm{~mm}^{2}$ ultra low adhesion plates (Corning). Between days 7 and 8, each EB was sandwiched between Matrigel drops (400 $\mu \mathrm{L}$ total) on a glass bottom dish (MatTek). Imaging of EB sprouting was performed over time (days 7, 10, 14, 18, and 21) using a Laser Scanning Confocal microscope (Technical Instruments).

\section{Differentiation of ESC into mesoderm}

A3 and B2 ESC were plated at 10,000 cells $/ \mathrm{cm}^{2}$ on $50 \mathrm{ng} / \mathrm{mL}$ of collagen IV-coated or fibronectin-coated plates (BD Biosciences) and cultured in Stage 1 Differentiation Medium, called NSD12b, for A3 and B2, respectively. ${ }^{24}$ Briefly, our serum-free Stage 1 Differentiation Medium, preoptimized for B2 and A3 cells included alpha-DMEM (Invitrogen), 15\% KSR (Invitrogen), 1x penicillin-streptomycin (Invitrogen), 1x nonessential amino acids (Invitrogen), $2 \mathrm{mM}$ L-glutamine (Invitrogen), $0.1 \mathrm{mM}$ 2-mercaptoethanol (Calbiochem), $5 \mathrm{ng} / \mathrm{mL}$ of bone morphogenetic protein-4 (R\&D Systems) and 0 or $20 \mathrm{ng} / \mathrm{mL}$ of VEGF (R\&D Systems) for A3 and B2, respectively. At 0, $1,2,3$, and 4 days, the cells were collected and stained for upregulation of mesoderm marker, Flk-1, and downregulation of pluripotent stem cell marker, Oct3/4. Briefly, cells were fixed 4\% paraformaldehyde overnight before being placed in a solution of $1 \%$ bovine serum albumin (BSA) (Sigma), 0.7\% Triton X-100 (MP Biomedicals LLC), and 5\% donkey serum (Fitzgerald) for 1 hour prior to staining. Cells were stained with Alexa Fluor 647 anti-mouse Flk-1 (Biolegend), or anti-Oct3/4 PE conjugated antibody (Becton Dickinson [BD]) overnight. Cells were rinsed and analyzed on an LSR II flow cytometer (BD). All results were gated to $5 \%$ of an isotype control.

\section{Fluorescence tracking during differentiation}

A3 mESC were plated at 10,000 cells $/ \mathrm{cm}^{2}$ on $50 \mathrm{ng} / \mathrm{mL}$ fibronectin-coated plates (BD Biosciences) and cultured in Stage 1 Differentiation Medium. ${ }^{24}$ After 3 days, the cells were replated at 10,000 cells $/ \mathrm{cm}^{2}$ on $50 \mathrm{ng} / \mathrm{mL}$ fibronectincoated plates and cultured in Stage 2 Differentiation Medium containing 70\% alpha-MEM (Mediatech) and 30\% DMEM (Invitrogen) plus 2x Nutridoma CS (Roche), 1x penicillinstreptomycin (Invitrogen), 1x nonessential amino acids (Invitrogen), $2 \mathrm{mM}$ L-glutamine (Invitrogen), and $0.05 \mathrm{mM}$ 2-mercaptoethanol (Calbiochem), $10 \mathrm{ng} / \mathrm{mL}$ basic Fibroblast 
Growth Factor (Sigma), and $10 \mathrm{ng} / \mathrm{mL}$ VEGF (R\&D Systems). On days $5,7,10,12$, and 14 of total differentiation, the differentiating cells were removed using Cell Dissociation Buffer (Invitrogen) for 30-60 minutes and analyzed for Tie-2 GFP or $\alpha$-SMA RFP expression on an Aria III Flow Cytometer (BD). Data analyses were performed using FlowJo software (Tree Star Inc.). FITC- and PE-labeled compensation beads were used for compensation of potentially double positive cells. Unstained and undifferentiated A3 mESC were used as a negative control and gated to $<5 \%$ of the negative control. Fluorescent populations of cells were analyzed for statistical significance using one-way analysis of variance and a post hoc Tukey's HSD test using the R programming language.

\section{Results}

\section{Characterization of $\mathrm{mESC}$}

Out of the original 6 blastocysts that were isolated from one pregnant mouse, 4 resulted in embryonic stem cell lines, that is, greater than 50\% efficiency (Figure 1). These exhibited typical 3-D colony-like morphology, expressed high levels of alkaline phosphatase activity (Figure 2A and B) and retained normal mouse karyotype of 40 chromosomes (Figure 2C and D).
The mESC lines also express pluripotent stem cell markers Oct 3/4 transcription factor (Figure 2E and F), stage-specific embryonic antigen-1 (Figure $2 \mathrm{G}$ and $\mathrm{H}$ ), as well as Sox-2 (Figure 2I and $\mathrm{J}$ ) and Nanog (Figure 2K and L).

\section{Mouse ESC generate cells from all three germ layers}

In order to verify the pluripotency of the new ESC lines, ESC were injected into the dorsal hind flank of NSG knockout mice to assay for teratoma formation. Differentiated tissue from both A3 and B2 mESC lines were able to form tissues from all three germ layers including digestive (Figure 3A) and secretory endoderm (Figure 3D), pacinian corpuscles indicating ectodermal lineage (Figure $3 \mathrm{~B}$ and $\mathrm{E}$ ), and an abundance of skeletal (Figure 3C) and cardiac muscle (Figure 3F) mesoderm tissue.

\section{Mouse ESC fluorescent markers express in the corresponding cell phenotype}

Within both tissues, Tie-2 GFP+ cells and $\alpha$-SMA $\mathrm{RFP}+$ cells were present and interacting with each other (Figure $3 \mathrm{G}$ and $\mathrm{H}$ ). In order to verify specificity of the
A3
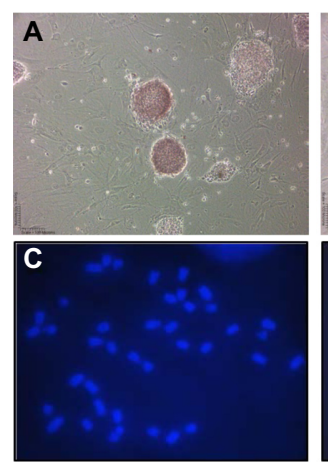

B2

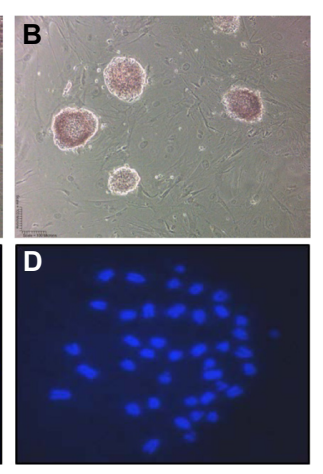

A3
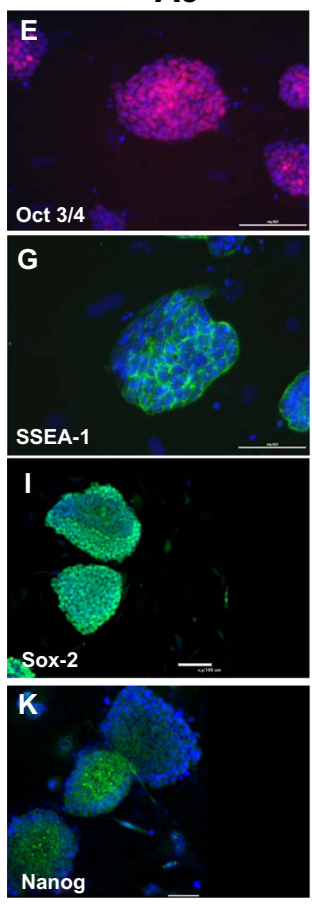

B2

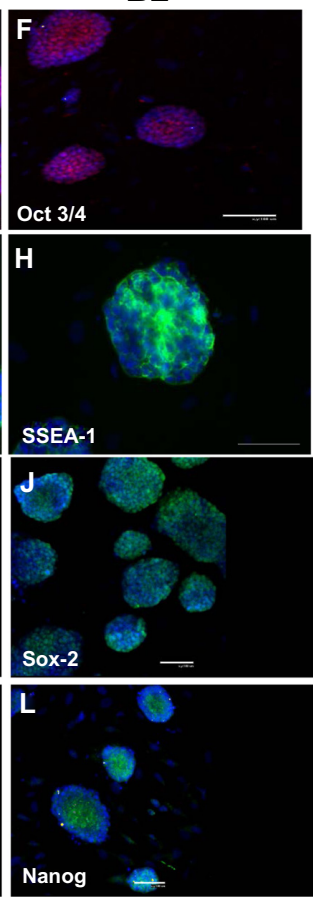

Figure 2 ESC stain positive for markers consistent with undifferentiated stem cells.

Notes: (A and B) The A3 and B2 ESC lines, respectively, display typical ESC colony morphology and display high levels of alkaline phosphatase activity. (C and D) A3 and B2 ESC cell lines display normal karyotypes with 40 chromosomes each. (E-L) A3 and B2 mESC display typical ESC colony morphology and markers: (E and F), Oct $3 / 4$ (red); (G and H), SSEA-I (green); (I and J), Sox-2 (green); and (K and L), Nanog (green), respectively. All cells are counterstained with DAPI. Scale bars =I00 $\mu$ m.

Abbreviations: DAPI, 4',6-diamidino-2-phenylindole; ESC, embryonic stem cells; SSEA-I, stage-specific embryonic antigen-I. 

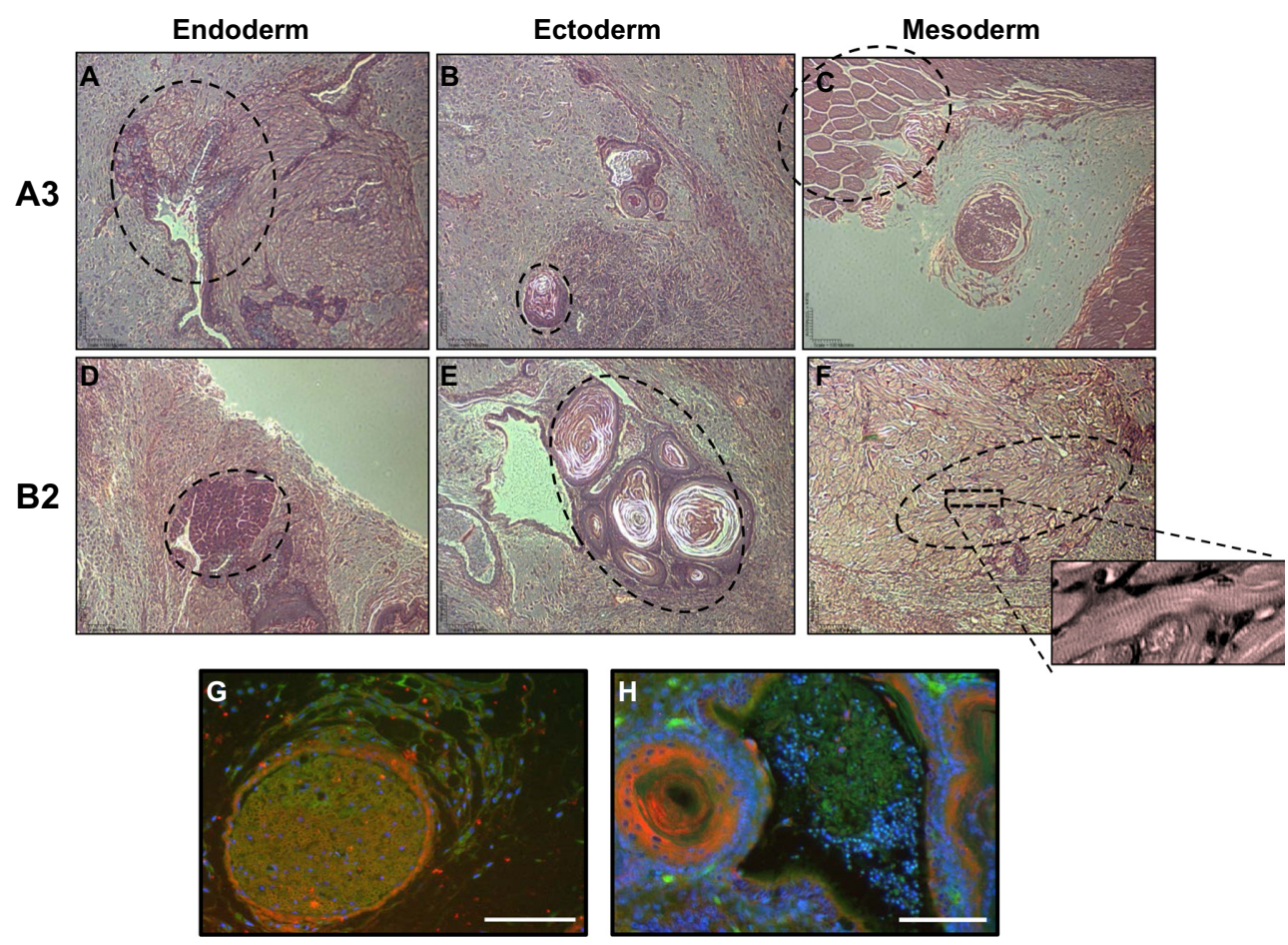

Figure $3 \mathrm{~A} 3$ and B2 ESC cell lines differentiate into cells from the three germ layers within teratomas.

Notes: Tumor assays of (A-C) A3-ESC exhibiting endoderm, ectoderm, and mesoderm, respectively. (D-F) B2-ESC exhibiting endoderm, ectoderm, and mesoderm, respectively. The selected A3-ESC images show (A) elongated columnar cells (acinar epithelium) with dark cytoplasm of the digestive organs. (B) The circular structure near the bottom is dermis sensory tissue consistent with the onion-like Pacinian corpuscle. (C) The circular structures closely resemble myofibrils of skeletal muscle. The B2-ESC developed (D) exocrine cells containing nuclei surrounded by small, cuboidal, uniformly dark stained cytoplasm. This is a defining characteristic of serous acini cells found in the secretory tissue. The B2-ESC tumors also developed (E) Pacinian corpuscles and (F) a region of cardiac muscle (dotted circle) with clearly defined sarcomere structures. Teratomas also display Tie-2 GFP+ and $\alpha$-SMA RFP+ cells interacting with each other. (G) A3-ESC formed tissue resembling a lymph follicle with developing cells weakly expressing Tie-2 GFP+ surrounded by $\alpha$-SMA RFP+ cells and strongly Tie-2 GFP+ cells that form venule-like structures. (H) B2-ESC form layers of $\alpha$-SMA RFP+ cells that appear to be similar to the dermis sensory tissue of a pacinian corpuscle and are infiltrated with Tie-2 GFP+ cells that are indicative of small blood vessels. Scale bars $=100 \mu \mathrm{m}$.

Abbreviations: $\alpha$-SMA, alpha-smooth muscle actin; ESC, embryonic stem cells; GFP, green fluorescent protein; RFP, red fluorescent protein.

reporter genes, the histology slides from the teratomas were stained for VE-cadherin or smooth muscle-myosin heavy chain (SM-MHC). Both the A3 and B2 cell lines exhibited the endothelial specific marker, VE-cadherin (Figure 4A and D), colocalized with Tie-2 GFP (Figure 4B and E). An overlay of the two images confirms dual expression (Figure $4 \mathrm{C}$ and $\mathrm{F}$ ). Similarly, the expression of the mature smooth muscle cell marker, SM-MHC, was detected (Figure 4G and J) with $\alpha$-SMA RFP expression (Figure 4H and $\mathrm{K}$ ). Merging of the images localizes expression to a strip of smooth muscle cells (Figure 4I) as well as a pacinian corpuscle (Figure 4L).

\section{Differentiated ESC generate vascular structures}

In order to validate that the new mESC lines expressed both reporters during differentiation, $\mathrm{EB}$ were generated from both mESC lines. The A3 stem cell line expressed the Tie-2 GFP fluorescent reporter as early as day 7 (Figure 5A) with interconnected networks at day 10 (Figure 5B). This is consistent with embryogenesis, where Tie- 2 is first detectable on day 8 , with widespread expression by day $9.5 .{ }^{20}$ Individual $\alpha$-SMA RFP+ cells were observed within sprouting EB at days 14 and 18 (Figure 5C and D, respectively, and Video S1A). In contrast, the B2 EB formed a large Tie-2-GFP+ networks at day 10 that were maintained through day 14 . By day 18 , the structures appear to have been remodeled into smaller capillary-like structures with more $\alpha$-SMA RFP+ cells surrounding the structures with some regression seen by day 21 (Figure 5E-H and Video S1B).

\section{Temporal development of mesoderm differentiating in monolayer cultures}

The differentiation of ESC into EC is performed in two distinct stages, as previously published ${ }^{24}$ : ESC differentiate into Flk-1+ progenitor cells in our Stage 1 Differentiation Medium, then the Flk-1+ cells are replated in Stage 2 Differentiation Medium (Figure S1). The vascular progenitor cell marker, Flk-1, expression for both the A3 and B2 

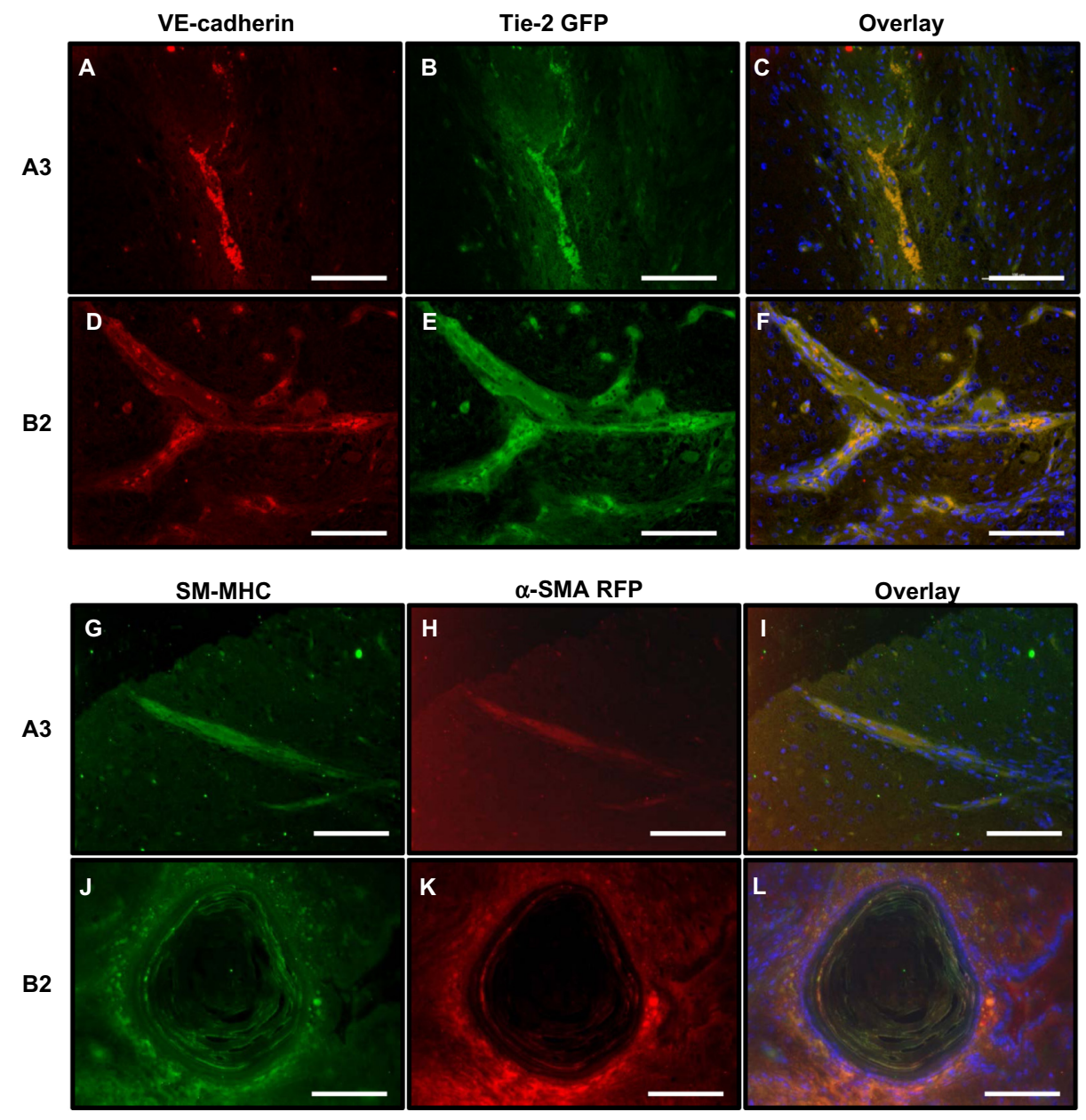

Figure $4 A 3$ and $B 2$ ESC cell lines coexpress fluorescent proteins and markers of mature $E C$ and SMC within teratomas.

Notes: (A-F) VE-cadherin+ (red) cells form vessel-like structures and colocalize with the Tie-2 GFP cells for A3 and B2 mESC lines. (G-L) Likewise, SM-MHC+ cells (green) are coexpressed with the $\alpha$-SMA RFP cells in both mESC lines. All cells were counterstained with DAPI (blue). Scale bars $=100 \mu \mathrm{m}$.

Abbreviations: $\alpha$-SMA, alpha-smooth muscle actin; GFP, green fluorescent protein; RFP, red fluorescent protein; SM-MHC, smooth muscle myosin heavy chain; VE, vascular endothelial; ESC, embryonic stem cells; mESC, mouse ESC; VE, vascular endothelial.
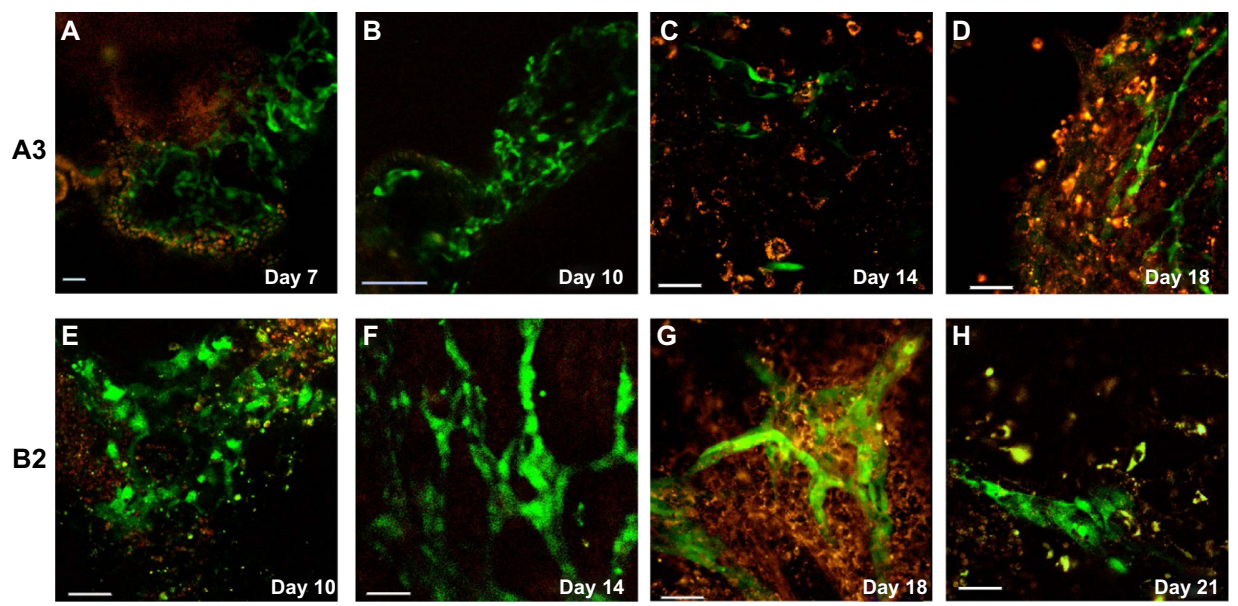

Figure 5 Embryoid bodies (EB) develop vascular Tie-2 GFP+ and $\alpha$-SMA RFP+ cells.

Notes: The differentiating EB of (A-D) A3-ESC and (E-H) B2-ESC from day 7 through 21. The Tie-2 expression under a GFP promoter is first observed at day 7 for A3 and day 10 for B2, followed by $\alpha$-SMA RFP cells surrounding the structures. Note that by day 18 and 21 for $A 3$ and $B 2$, respectively, the larger vessel-like structures appear to be remodeled into smaller, more compact vessel structures. The subtle differences in expression patterns are most likely due to inherent differences between the $\mathrm{mESC}$ lines. Scale bars $=50 \mu \mathrm{m}$.

Abbreviations: $\alpha$-SMA, alpha-smooth muscle actin; ESC, embryonic stem cells; GFP, green fluorescent protein; mESC, mouse embryonic stem cell; RFP, red fluorescent protein. 
A

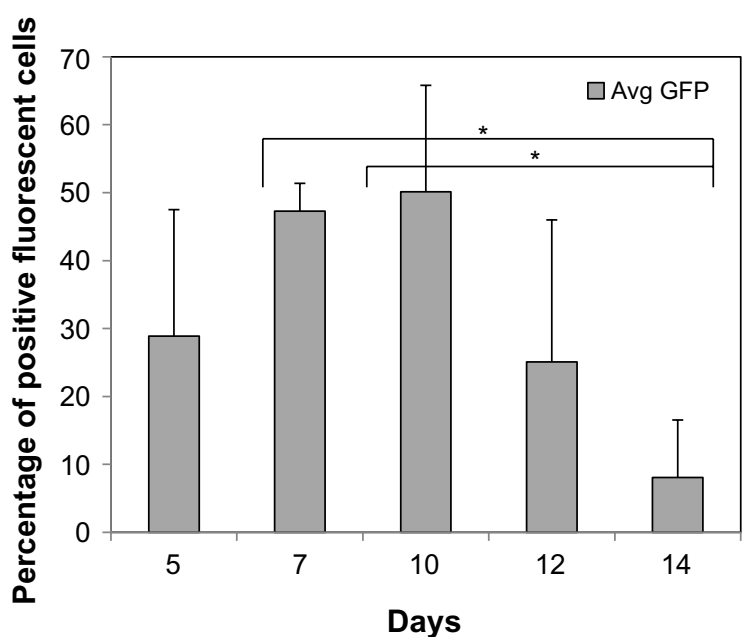

B

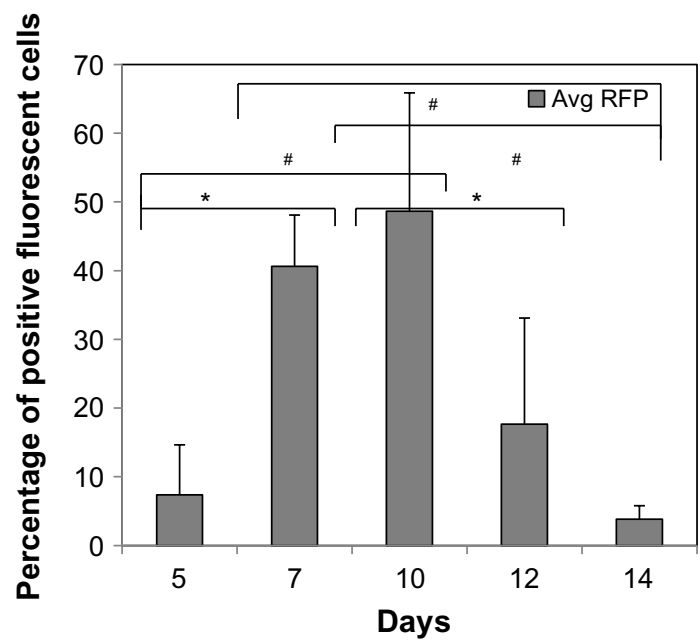

Figure 6 Quantification of temporal expression of Tie-2 GFP+ and $\alpha$-SMA RFP+ cells differentiating as monolayer cultures.

Notes: The Flk-I+ cells were re-plated as monolayers and allowed to differentiate overtime. The (A) Tie-2 GFP+ and (B) $\alpha$-SMA RFP+ cells were then quantified as a function of time. Note that the first observed expression is seen at day 5 , slightly earlier than the EB differentiation methods. ${ }^{*} P<0.05$ and ${ }^{\# P}<0.0 \mathrm{I}, \mathrm{N} \geq 3$ distinct experiments.

Abbreviations: Avg, average; $\alpha$-SMA, alpha-smooth muscle actin; GFP, green fluorescent protein; RFP, red fluorescent protein.

ESC lines was followed over an initial 4 days. Both cell lines expressed the greatest percentage of Flk-1+ cells at day 3 of induction (Figure S2). The upregulation of Flk-1+ cells also coincided with a corresponding decrease in Oct 3/4 expression (Figure S2). Using Stage 2 Differentiation Medium, we then examined the GFP and RFP expression of differentiating A3-ESC over time (Figure 6A and B). The expression of both of these Tie-2 GFP+ and $\alpha$-SMA RFP+ vascular cells was first observed in monolayer cultures and then quantified by fluorescence activated cell sorting analysis from day 5 (2 days after plating the Flk-1+ cells) through day 14 of total differentiation. The Tie-2 GFP+ cells were first observed at day 5, slightly earlier than the 3-D EB method of differentiation in which the Tie-2+ vascular-like structures first emerged at day 7 . The $\alpha$-SMA RFP+ was also observed on the early mesodermal cells, peaking at day 10 , the same time as the Tie-2 GFP+ cells.

\section{Conclusion}

In these mouse ESC lines, the expression of GFP indicates EC commitment while RFP identifies the emerging smooth muscle cells. To our knowledge, there is not another ESC line that currently identifies both vascular EC and SMC. This unique system was specifically designed to allow realtime studies of vascular stem cell fate from stem cell populations or single cells because these cells do not require fixation in order to track their developmental progress. The RFP expression in SMC, which do not express unique cell surface markers, may also provide an additional "handle" for fluorescence activated cell sorting SMC from other differentiating cell types. Our 2-D and 3-D differentiation systems both show expression of Tie-2+ and $\alpha$-SMA+ cells at day 7 and increase up to days 18 to 21 of differentiation, indicating a closely related temporal development.

It is also important to note that these markers are excellent, but still not completely definitive for endothelial and vascular smooth muscle. The $\alpha$-SMA is also expressed in early embryonic cardiac cells and Tie-2 is expressed on hematopoietic cells. Therefore, the differentiation and isolation of these cells was monitored in conjunction with morphology and/or differentiation kinetics of the system. Despite this drawback, these cells provide an improved model for directing vascular fate in conjunction with morphological development of the vasculature in a 3-D system.

\section{Acknowledgments}

This work was funded, in part, through an National Science Foundation Integrative Graduate Education and Research Traineeship Award \# 0965918, NSF-Science and Technology Center for the Emergent Behavior of Integrated Biological Systems Award \# 0939511, and a New Faculty Award from the California Institute for Regenerative Medicine Award \# RN2-00921-1. We also thank Yunzhe Zhang for her technical assistance with ESC karyotyping.

\section{Disclosures}

The authors report no conflicts of interest in this work. 


\section{References}

1. Amit M, Carpenter MK, Inokuma MS, et al. Clonally derived human embryonic stem cell lines maintain pluripotency and proliferative potential for prolonged periods of culture. Dev Biol. 2000;227:271-278.

2. Kalka C, Masuda H, Takahashi T, et al. Transplantation of ex vivo expanded endothelial progenitor cells for therapeutic neovascularization. Proc Natl Acad Sci U S A. 2000;97:3422-3427.

3. Kaushal S, Amiel GE, Guleserian KJ, et al. Functional small-diameter neovessels created using endothelial progenitor cells expanded ex vivo. Nat Med. 2001;7:1035-1040.

4. Griese DP, Ehsan A, Melo LG, et al. Isolation and transplantation of autologous circulating endothelial cells into denuded vessels and prosthetic grafts: implications for cell-based vascular therapy. Circulation. 2003;108:2710-2715.

5. Li Z, Han Z, Wu JC. Transplantation of human embryonic stem cell-derived endothelial cells for vascular diseases. J Cell Biochem. 2009;106:194-199.

6. Flamme I, Frolich T, Risau W. Molecular mechanisms of vasculogenesis and embryonic angiogenesis. J Cell Physiol. 1997;173:206-210.

7. Yamaguchi TP, Dumont DJ, Conlon RA, Breitman ML, Rossant J. Flk-1, an flt-related receptor tyrosine kinase is an early marker for endothelial cell precursors. Development. 1993;118:489-498.

8. Eichmann A, Corbel C, Nataf V, Vaigot P, Bréant C, Le Douarin NM. Ligand-dependent development of the endothelial and hemopoietic lineages from embryonic mesodermal cells expressing vascular endothelial growth factor receptor 2. Proc Natl Acad Sci USA. 1997;94: 5141-5146.

9. Hirashima M, Kataoka H, Nishikawa S, Matsuyoshi N, Nishikawa S. Maturation of embryonic stem cells into endothelial cells in an in vitro model of vasculogenesis. Blood. 1999;93:1253-1263.

10. Nishikawa SI, Nishikawa S, Hirashima M, Matsuyoshi N, Kodama H. Progressive lineage analysis by cell sorting and culture identifies FLK1+VE-cadherin+ cells at a diverging point of endothelial and hemopoietic lineages. Development. 1998;125:1747-1757.

11. Shalaby F, Rossant J, Yamaguchi TP, et al. Failure of blood-island formation and vasculogenesis in Flk-1-deficient mice. Nature. 1995;376:62-66.

12. Yamashita J, Itoh H, Hirashima M, et al. Flk1-positive cells derived from embryonic stem cells serve as vascular progenitors. Nature. 2000;408:92-96.
13. Park C, Afrikanova I, Chung YS, et al. A hierarchical order of factors in the generation of FLK1-and SCL-expressing hematopoietic and endothelial progenitors from embryonic stem cells. Development. 2004;131:2749-2762.

14. Yamashita JK. Differentiation of arterial, venous, and lymphatic endothelial cells from vascular progenitors. Trends Cardiovasc Med. 2007;17:59-63.

15. Battista S, Guarnieri D, Borselli C, et al. The effect of matrix composition of $3 \mathrm{D}$ constructs on embryonic stem cell differentiation. Biomaterials. 2005;26:6194-6207.

16. Flaim CJ, Chien S, Bhatia SN. An extracellular matrix microarray for probing cellular differentiation. Nat Methods. 2005;2:119-125.

17. Takakura N, Huang XL, Naruse T, et al. Critical role of the TIE2 endothelial cell receptor in the development of definitive hematopoiesis. Immunity. 1998;9:677-686.

18. Suri C, Jones PF, Patan S, et al. Requisite role of angiopoietin-1, a ligand for the TIE2 receptor, during embryonic angiogenesis. Cell. 1996;87: 1171-1180.

19. Magness ST, Bataller R, Yang L, Brenner DA. A dual reporter gene transgenic mouse demonstrates heterogeneity in hepatic fibrogenic cell populations. Hepatology. 2004;40:1151-1159.

20. Motoike T, Loughna S, Perens E, et al. Universal GFP reporter for the study of vascular development. Genesis. 2000;28:75-81.

21. Evans MJ, Kaufman MH. Establishment in culture of pluripotential cells from mouse embryos. Nature. 1981;292:154-156.

22. Evans SM. Isolation and maintenance of murine embryonic stem cells. In: Lanza R, editor. Handbook of Stem Cells. Vol 1. Boston MA: Elsevier Academic; 2004:413-417.

23. Hunter SM, Evans M. Non-surgical method for the inductions of delayed implantation and recovery of viable blastocysts in rats and mice by the use of tamoxifen and Depo-Provera. Mol Reprod Dev. 1999;52:29-32.

24. Blancas A, Shih AJ, Lauer NE, McCloskey KE. Endothelial cells from embryonic stem cells in a chemically defined medium. Stem Cells Dev. 2011;20:2153-2161. 


\section{Supplementary materials}

Video SI The videos contain the Z-stacked images of the developing vascular structures within embryoid bodies from (A) A3 and (B) B2 ESC, respectively.

Abbreviation: ESC, embryonic stem cells.

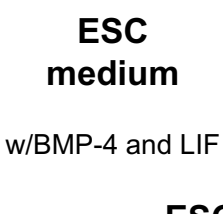

ESC

\section{Differentiating ESC}

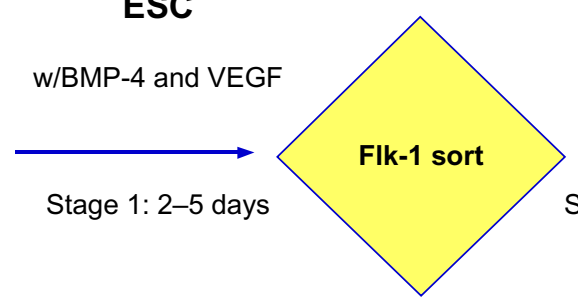

Flk-1+ outgrowths

w/VEGF and bFGF

Stage 2: up to 14 days

Figure SI Flow chart of the stage-specific differentiation used to obtain mature ESC-EC in chemically defined conditions.

Notes: Stage I was optimized for the induction of ESC towards mesoderm, specifically Flk-I+ vascular progenitor cells. Stage 2 induces further differentiation of the Flk- I+ cells into vascular cell derivatives.

Abbreviations: bFGF, basic fibroblast growth factor; BMP-4, bone morphogenetic protein-4; EC, endothelial cell; ESC, embryonic stem cells; LIF, leukemia inhibitory factor; VEGF, vascular endothelial growth factor; w/, with.

A3

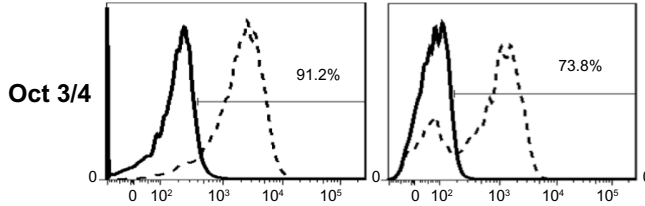

Day 0

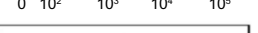

Flk-1

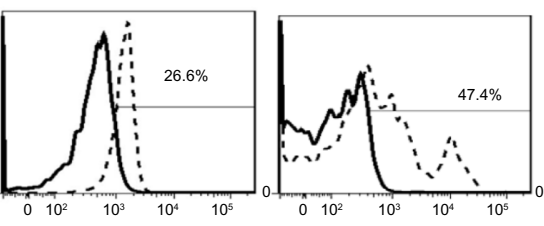

B2
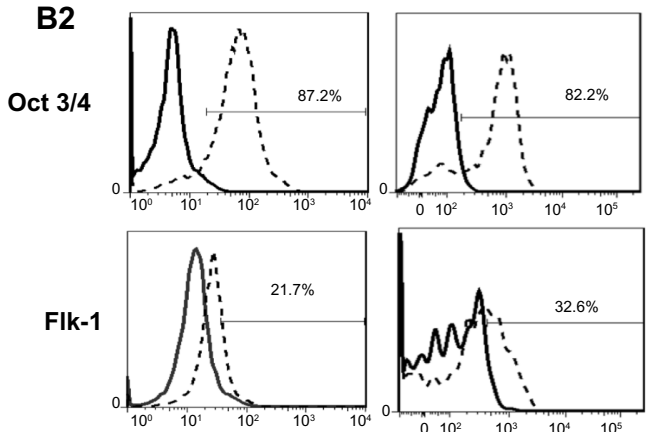

Day 2
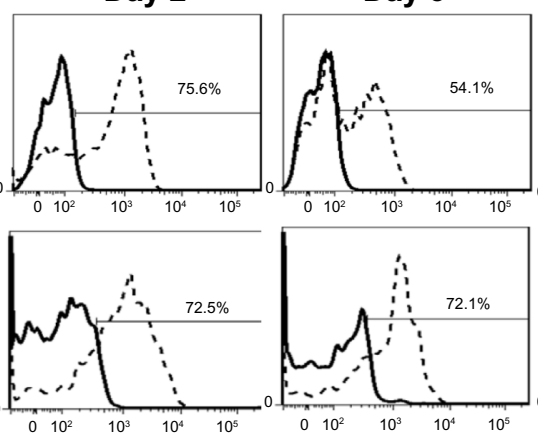

Day 3
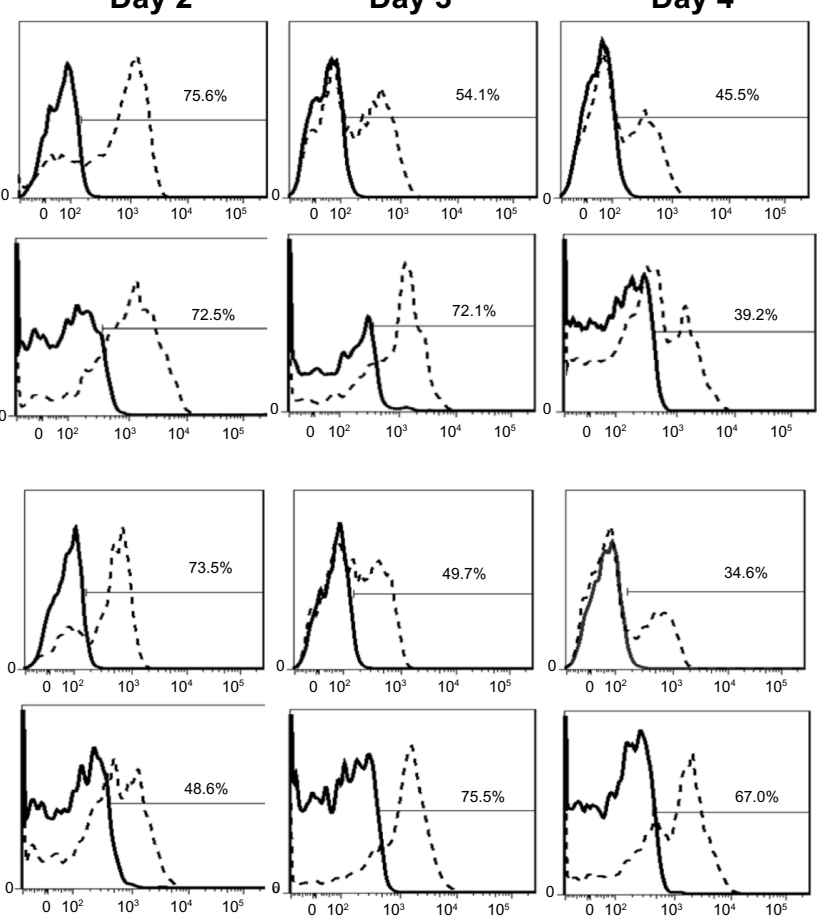

Figure S2 The ESC marker Oct 3/4 declines as ESC differentiate.

Note: Under optimal conditions for differentiation of ESC to Flk-I+ cells, Oct 3/4 undergoes a marked decrease from day 2 to day 3 , as Flk-I expression peaks and is sustained in both $\mathrm{A} 3$ and $\mathrm{B} 2 \mathrm{mESC}$.

Abbreviations: ESC, embryonic stem cells, mESC, mouse ESC.

Stem Cells and Cloning: Advances and Applications

\section{Publish your work in this journal}

Stem Cells and Cloning: Advances and Applications is an international, peer-reviewed, open access journal. Areas of interest in stem cell research include: Embryonic stem cells; Adult stem cells; Blastocysts; Cordblood stem cells; Stem cell transformation and culture; Therapeutic cloning; Umbilical cord blood and bone marrow cells; Laboratory,

\section{Dovepress}

animal and human therapeutic studies; Philosophical and ethical issues related to stem cell research. This journal is indexed on CAS. The manuscript management system is completely online and includes a quick and fair peer-review system. Visit http://www.dovepress.com/ testimonials.php to read real quotes from published authors. 